(C) [2007] IEEE. Reprinted, with permission, from [Mo Li, Kumbesan Sandrasegaran and Tracy Tung,A MultiInterface Proposal for IEEE 802.21 Media Independent Handover, Management of Mobile Business, 2007. ICMB 2007. International Conference on July 2007]. This material is posted here with permission of the IEEE. Such ermission of the IEEE does not in any way imply IEEE endorsement of any of the University of Technology, Sydney's products or services. Internal or personal use of this material is permitted. However, permission to reprint/republish this material for advertising or promotional purposes or for creating new collective works for resale or redistribution must be obtained from the IEEE by writing to pubs-permissions@ieee.org. By choosing to view this document, you agree to all provisions of the copyright laws protecting it 


\title{
A Multi-Interface Proposal for IEEE 802.21 Media Independent Handover
}

\author{
Mo Li, Kumbesan Sandrasegaran and Tracy Tung \\ Institute of Information and Communication Technologies and Faculty of Engineering \\ University of Technology, Sydney \\ Email: \{moli, kumbes, ttung\}@eng.uts.edu.au
}

\begin{abstract}
In Next-Generation (NG) wireless networks, hybrid handover techniques are expected to enable the integration of heterogeneous networks, e.g. 3G cellular networks and 802.11 Wireless LAN (WLAN). Media Independent Handover (MIH) has begun to be discussed in the latest IEEE 802.21 to accommodate multiple disparate interfaces in mobile handset. Multiple interfaces can come in a variety of ways to facilitate hybrid handover, and may result in different requirements on protocols and performance. In this paper, we classify multi-interface schemes for handover in heterogeneous wireless networks. We propose a multi-interface scheme for IEEE 802.21 MIH. The scheme is proposed to be able to work with standard TCP and Mobile IPv4 agent routers without particular configuration. Based on this scheme, we implemented a dual-interface Mobile Host (MH) model in Network Simulation 2 (ns2) to evaluate how it performs in comparison with single-interface $\mathrm{MH}$.
\end{abstract}

\section{Introduction}

In Next-Generation (NG) wireless networks, hybrid handover techniques are expected to enable the integration of heterogeneous networks, e.g. 3G cellular networks and 802.11 Wireless LAN (WLAN). Hybrid handover is the process, in which Mobile Host (MH) is switched between domains of different access technologies, or between domains of multiple operators.

Figure 1 illustrates the procedures involved in a hybrid handover in the integration of 3G Public Land Mobile Network (PLMN) and WLAN. Generally, hybrid handover involves network selection from a list of discovered Point of Attachments (POA). The Mobile Host's (MH) association with newly selected POA can be established while keeping an ongoing session with the current POA. To support hybrid handover, $\mathrm{MH}$ must have multiple network interfaces for accessing disparate networks. The multi-interface solutions of $\mathrm{MH}$ have been studied in a number of papers [1, 2]. Single-interface solutions for accessing multiple networks were also addressed in $[3,4]$. In hybrid handover, dealing with the heterogeneities of multiple access technologies in a consistent manner would be a challenge for multi-interface design. Media Independent Handover (MIH) was recently proposed in IEEE 802.21 for discussion. The IEEE 802.21 [5] is a developing effort to enable handover and interoperability between heterogeneous networks.

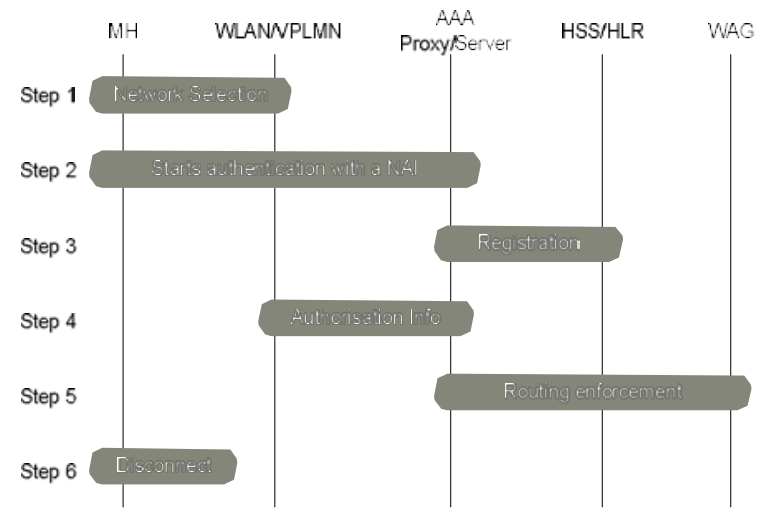

Figure 1 Handover Procedures in the Integration of 3G PLMN and WLAN networks

IEEE 802.21 framework defines three different services to facilitate information exchange for network discovery and selection in hybrid handover. Media Independent Event Service (MIES) provides events notification in response to changes in network conditions. Media Independent Command Service (MICS) is used to manage and control link behavior relevant to handover. Media Independent Information Service (MIIS) provides a model for information gathering.

Although IEEE 802.21 MIH defines an effective framework for enabling multiple disparate interfaces in mobile handset, the different implementation may result in varied performance in handover. The multiple interfaces can be simultaneously used to facilitate the so-called soft handover, which requires corresponding 
processing at upper layers. While, in another approach of hard handover, multiple interfaces can be alternatively used, and thus keep compatible with network layer protocols being widely used. In this context, we propose a multi-interface scheme for 802.21 MIH, and evaluate its handover performance on Network Simulation 2 (ns2) [6].

The rest of the paper is organized as follows. The multi-interface schemes of $\mathrm{MH}$ for hybrid handover are classified in Section 2. Section 3 describes a multiinterface scheme proposed for 802.21 MIH. In section 4 , we introduce the implementation of dual-interface $\mathrm{MH}$ model in ns2. The simulation scenario and experimental results are demonstrated in Section 5. We conclude the paper in Section 6.

\section{Multi-Access Schemes}

Multiple interfaces in mobile handset can come in a variety of ways to support accessing heterogeneous wireless networks. Some selective terms on multiinterface design are explained as follows:

- Simultaneous communication: refers to the ability of having multiple interfaces carry data communication simultaneously.

- Address management: host mobility management has been addressed in both Mobile IPv4 [7] and Mobile IPv6 [8]. MH can acquire multiple Care of Address (CoA) for accessing multiple visited domains. The related IP address assignment on each interface is referred as address management.

- Traffic redirection: the ongoing sessions/traffic can be redirected from one interface to another due to handover.

- Network selection: is the process of selecting next POA with handover decision algorithms.

In this section, we classify and compare multiinterface schemes for accessing heterogeneous wireless networks. In addition, we also demonstrate a singleinterface scheme for accessing different network domains, which may belong to multiple operators.

\subsection{Multi-Interface Simultaneously Used}

Mobile handset may use multiple interfaces simultaneously to support a soft handover, which means $\mathrm{MH}$ always keeps at least one radio connection with POAs. The corresponding handover allows more than one interface to carry traffic flow so as to redirect ongoing sessions seamlessly. Mobile handset with multiple IP addresses is referred to as multihomed [9]. Because data stream would be carried through multiple network domains, multihoming solutions need support at both network and transport layers. Mobile IPv6 provides necessary mechanisms for multi-interface related address management and the corresponding solutions on nomadism of users for IP layer services. In [10], a number of transport multihoming protocols have been addressed. Basically, the transport protocol in multihoming should be able to deal with the packets originating from the same session being multicast on multiple paths. Additional signaling and data traffic would be loaded on networks. The problem with this scheme is its incompatibility with TCP, which has huge user base. This scheme requires the multihoming support in visited agent routers as well, which may not be available in heterogeneous environments.

\subsection{Multi-Interface Alternatively Used}

Multiple interfaces can also be alternatively used to support hard handover in heterogeneous networks, which is also known as "break before make". Because only one interface gets involved in data transmission, lower layer interface switching can be made transparent to upper layers, e.g. TCP. Multiple interfaces can be integrated for ubiquitous access, but may result in a longer handover delay in data transmission due to excess time required for setting up new connections. The network interfaces other than the one carrying traffic can be used for network discovery, and gathering information on potential POAs. Multiinterface alternatively used scheme has less traffic burden on networks than the simultaneously used multi-interface scheme. This scheme still uses mobile IP protocols for address management and location services. However, it doesn't require the mechanisms, such as multiple CoAs' binding, which may need special setting in home agent. Morever, no particular configuration is need in agent routers. This scheme can work well even in standard Mobile IPv4.

\subsection{Single Interface for Multiple Access}

Single network interface is used for accessing one specific network domain in most cases. However, under some circumstances, single network interface, e.g. IEEE 802.11, can be multiplexed to simultaneously connect to multiple network domains. In the MultiNet scheme proposed in [3], single IEEE 802.11 wireless card can support infrastructure and adhoc mode of communications at the same time. The basic idea behind the MultiNet is to multiplex wireless network card across multiple wireless networks. MultiNet-enabled mobile handset maintains several virtual adapters in its network stack, which act as virtual wireless interfaces. Another scheme called SyncScan [4] explores the freedom of beacon sending time to synchronize wireless node with the timing of AP beacons. SyncScan aims to reduce probing delay in 
scanning multiple channels, and has the potential to track beacons from nearby APs while carrying on communication with the current AP. But, similar to MultiNet, it also requires synchronization with networks and modification to network protocol stack.

\section{A Multi-Interface Scheme Proposed for Media Independent Handover (MIH)}

To support handover in heterogeneous wireless networks, we propose a multi-interface scheme that is compatible with the transport protocols already in use. It is based on the second multi-access scheme aforementioned in Sec. 2.2. The proposed scheme supports network interface selection, and is one implementation of the IEEE 802.21 framework [5] still in discussion. The scheme adopts the concept of crosslayer design, and proposes an intermediate layer that gathers information through different network interfaces to assist handover decision making. All the functions with the intermediate layer are implemented in a Handover Management Module (HMM). The HMM is proposed to make upper layers independent of the heterogeneities of multiple network interfaces. Some handover decision algorithms for hybrid handover, such as policy-enabled handoff [11] and AHP network selection [12], can be implemented in HMM to assist handover decision making. The communication between HMM and other layers can use 802.21 MIH defined services. Each network interface can work in either primary mode or standby mode. However, only the interface in primary mode is activated for carrying traffic streams as well as signaling. Other interfaces in standby mode are only allowed to receive signaling from new POAs.

Figure 2 illustrates the main functional components and their interaction with the HMM. The HMM obtain handover metrics information, such as signal strength, QoS and service type, from both lower layers and upper layers using "HMM Metrics" services. The "HMM Metrics" services are the implementation of 802.21 MIH Information/Event services of information gathering and event notification. To trigger interface switching after decision making, the HMM sends "HMM commands" (implementing 802.21 MIH Command Services) to both upper layers and lower layers. The corresponding operations in different layers would be followed. The HMM commands for lower layers are used to activate or deactivate network interfaces for interface switching. To achieve a fast handover, the HMM can also inform upper layers of any changes made to physical connections. For example, in 802.11 WLAN, when MH activates a standby interface for attachment to a new POA, the
HMM can send up HMM commands to mobile IP layer to start registration/solicitation immediately so as to avoid waiting time for next advertisement's arrival.

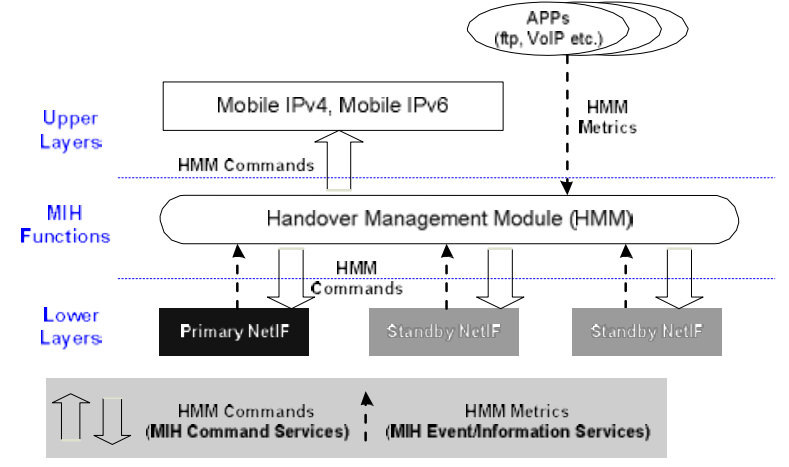

Figure 2 A Multi-Interface Scheme Proposed for 802.21 MIH

\section{Implementation Issues}

Based on the proposed multi-interface scheme shown in Figure 2, we implemented a dual-interface $\mathrm{MH}$ model in ns2 [6]. In this model, the HMM functions are implemented in a Handover Manager (HOMgr). We assume $\mathrm{MH}$ is equipped with two IEEE 802.11 interfaces. Its structure is illustrated in Figure 3. The simulation was developed on ns 2.29, with the following modifications:

- Support for multiple wireless channels on MH;

- Handover metrics probing at lower layer objects for information gathering;

- Modification to NO Ad-Hoc Routing Agent (NOAH) to be used as the routing agent for dualinterface $\mathrm{MH}$;

- Proactive triggering mechanisms in mobile IP protocol.

Because ns2 lacks the implementation of dealing with multiple interfaces, we also developed the following new components/functions for controlling and managing multiple interfaces:

- Handover manager with handover decision algorithms;

- Functions for network interface switching;

In dual-interface $\mathrm{MH}$. We use the $\mathrm{NOAH}$ [13] to direct communication between $\mathrm{MH}$ and APs. Two 802.11 interfaces have been tuned to different wireless channels, and both of them interact with the HOMgr. Mobile IPv4 was introduced to test MH's compatibility with legacy IP networks. Foreign Agent (FA) Care-ofAddress (CoA) is enabled on both interfaces when connecting to visited networks. Dual-interface $\mathrm{MH}$ is implemented such that: at anytime if the standby interface is activated, then the primary interface goes into standby mode. This would be followed by the 
termination of the data traffic on the previously activated interface. Upper layers (TCP/Applications) stay unknown to what happened at lower layers. HOMgr can regularly retrieve metric information through "Metric Probe" process, which is embedded at different layers. In the current implementation of HOMgr, we apply the signal power based handover decision algorithm [14].

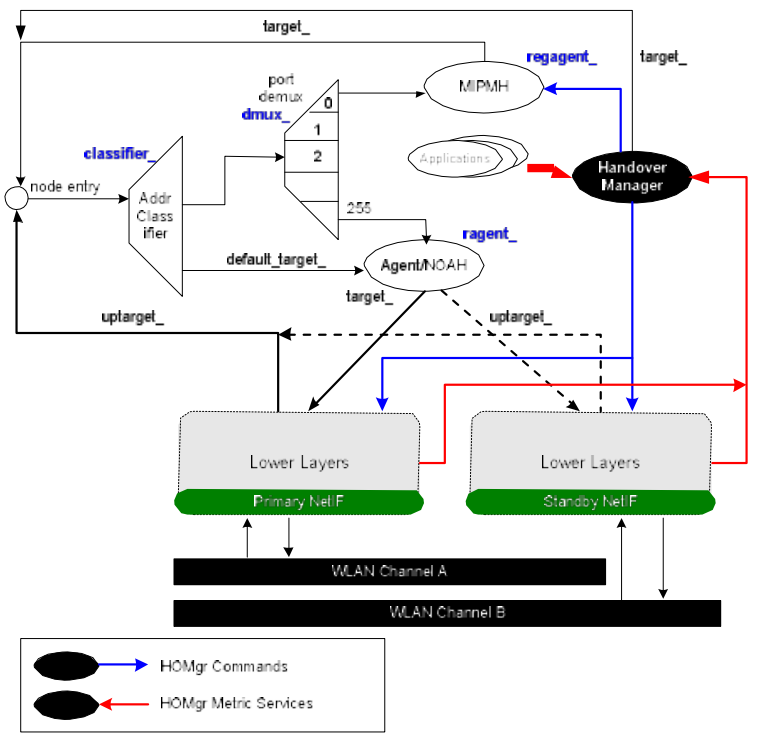

Figure 3 Dual-Interface Implementation in ns2

In implementation, we assume that $\mathrm{MH}$ skips channel scanning process in finding new POAs, which has been well studied in [4]. Authentication is also not implemented for handover association. HOMgr based multi-interface architecture can well accommodate other interface modules, such as GPRS and UMTS.

\section{Simulation and Results}

\section{A. Simulated Scenario}

In the simulated scenario, the Correspondent Node (CN) is connected to two separated 802.11 WLAN domains through a fixed link of 5Mbps. The AP of Home Agent (HA) is denoted as AP-HA, and AP-FA refers to the AP of Foreign Agent (FA). AP-HA and AP-FA are tuned to different wireless channels. Their radio coverage is made partially overlapped. As illustrated in Figure 4, MH was initially moving from AP-HA towards AP-FA at the speed of $20 \mathrm{~m} / \mathrm{s}$, and then went back to AP-HA.

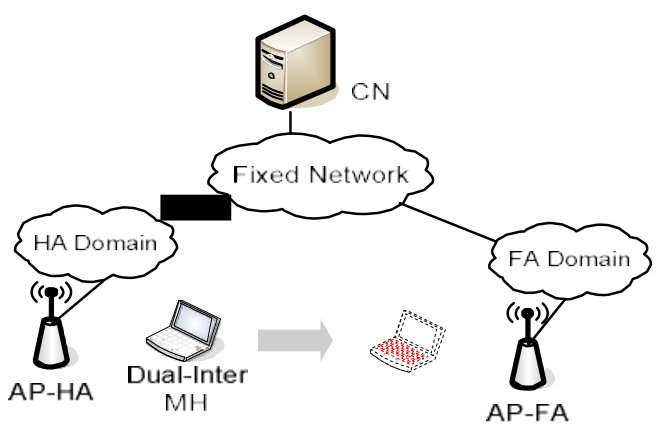

Figure 4 Wireless Overlap Scenario under Study

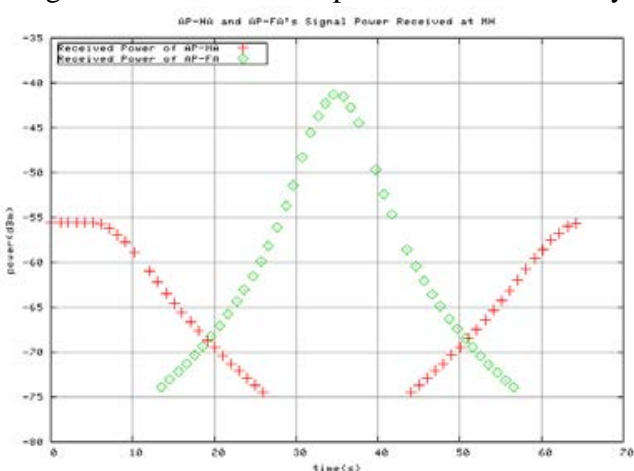

Figure 5 Received Signal Power at $\mathrm{MH}$

The radio coverage of AP-HA and AP-FA is a circle of $450 \mathrm{~m}$ in radius. TCP is the transport protocol applied between network nodes, through which constant bit rate (CBR) traffic is carried at the rate of $672 \mathrm{Kbps}$ (a 210-byte packet is sent every $2.25 \mathrm{~ms}$ ). Two handover events expected in this scenario are HAÆFA and FAÆHA respectively. The scenario of Figure 4 would be run for both dual-interface and single-interface $\mathrm{MH}$ so as to get a comparison result. Figure 5 shows the received signal power at $\mathrm{MH}$ for AP-HA and AP-FA.

\section{B. Simulation Results}

To evaluate the performance of the proposed multiinterface scheme, we simulated the above scenario on ns2 for both dual-interface $\mathrm{MH}$ and single-interface MH scenario. The simulation results of dual-interface $\mathrm{MH}$ would be compared with that of single-interface in the following three aspects.

\section{- Data Throughput}

The data throughput of dual-interface and singleinterface $\mathrm{MH}$ in the simulation is demonstrated in Figure 6. Both dual-interface and single-interface $\mathrm{MH}$ show common data transmission characteristics in homogeneous domains (non radio overlap areas). In the overlap areas, the dual-interface $\mathrm{MH}$ was able to conduct a faster handover to associate with new POA than the single-interface $\mathrm{MH}$. $\mathrm{MH}$ was set to allow the 
missing of just two consecutive advertisements. For single-interface $\mathrm{MH}$, the break time of TCP data transmission in both directions is 5.14s for "HAÆ FA" and 2.64s for "FAÆHA" respectively. In comparison, the dual-interface $\mathrm{MH}$ took just $67 \mathrm{~ms}$ to recover data transmission through visited FA in a handover, and $547 \mathrm{~ms}$ to have data transmission handed back to HA.

- End-to-end Packet Delay
The end-to-end packet delay is defined as the packet traveling time from traffic source $(\mathrm{CN})$ to traffic destination $(\mathrm{MH})$ for each confirmed TCP packet. From Figure 7, we noticed that the end-to-end packet delay during handover is in proportion to transmission breaking time. In the dual-interface scenario, handover still caused longer end-to-end packet delay compared with normal packet delivering time. However, the delay has been noticeably reduced to $700 \mathrm{~ms}$ if dualinterface is enabled on $\mathrm{MH}$, in comparison to over $5 \mathrm{~s}$ delay for single-interface $\mathrm{MH}$.

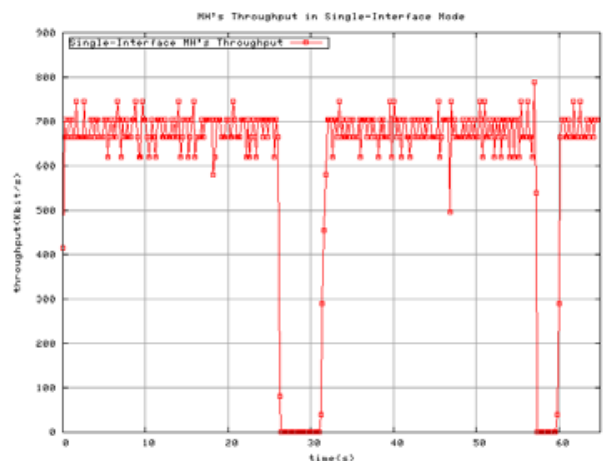

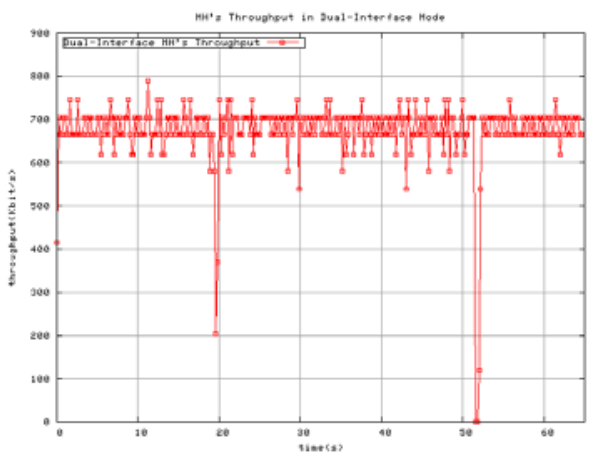

Figure 6 MH's Throughput (Dual-Interface vs. Single-Interface)
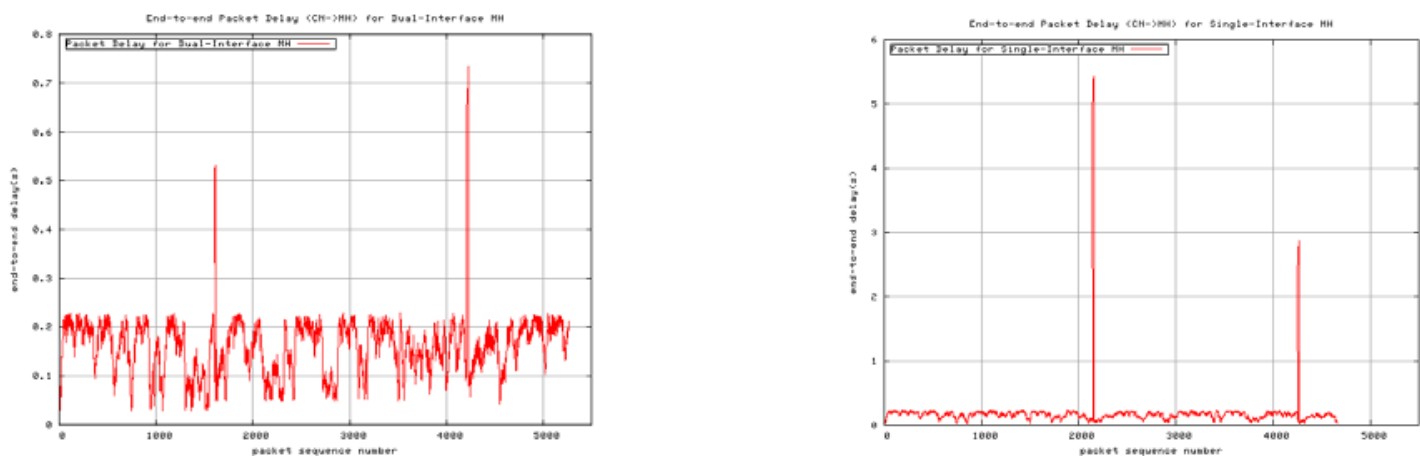

Figure 7 End-to-end Packet Delay from CN to MH (Dual-interface vs. Single-Interface)
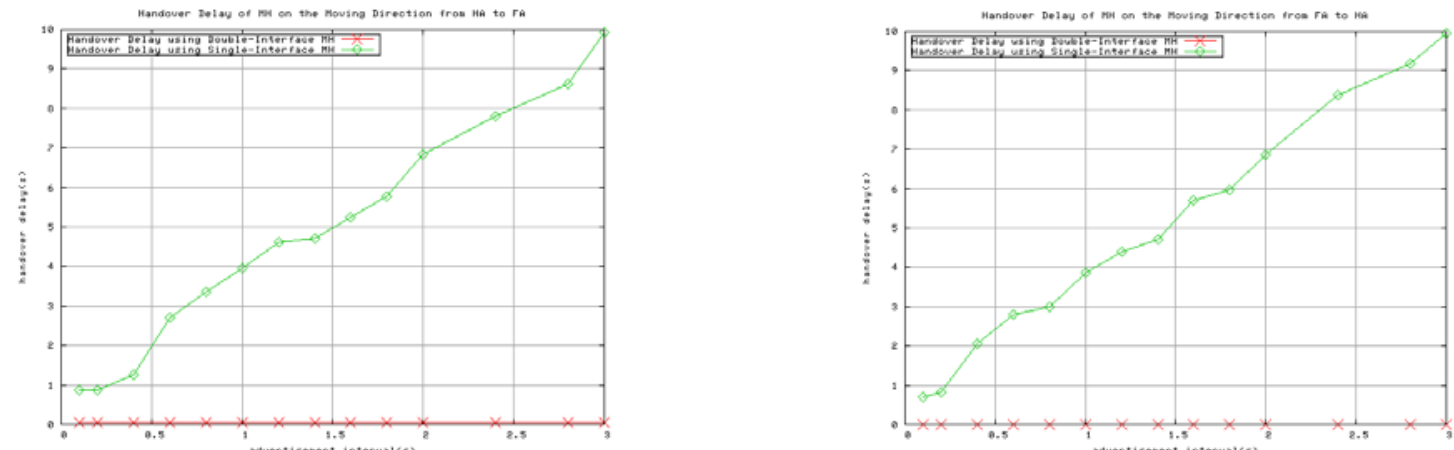

Figure 8 Handover Delay of MH with varying Advertisement Interval (HAÆFA vs. FAÆHA) 
- Handover Delay

Handover delay is defined as the time interval between the correctly receiving of the last packet from old POA and the completion of association with new POA. In the simulation, we set the agent advertisement (ads) interval ( $\mathrm{I}_{\text {ads }}$ ) to be one-third of the advertisement lifetime ( $\mathrm{L}_{\mathrm{ads}}$ ) according to [7]. We measured handover delay incurred in both movement directions of $\mathrm{MH}$. $\mathrm{I}_{\text {ads }}$ was increased in the simulation, and thus its impact on handover delay can be evaluated. The simulation results show that single-interface $\mathrm{MH}$ generally took much longer time to complete a handover than dual-interface $\mathrm{MH}$. Single-interface $\mathrm{MH}$ always waits until the ads from the currently attached AP expire before trying to find a new AP. In the simulation, an average $0.84 \mathrm{~s}$ handover delay was seen when $\mathrm{I}_{\mathrm{ads}}=0.2$. While, when $I_{\text {ads }}$ was 1s, handover delay reached 3.91s. For dual-interface $\mathrm{MH}$, handover delay was apparently independent of $\mathrm{I}_{\text {ads }}$ as demonstrated in Figure 8. The dual-interface is able to deal with handover in an proactive way, and thus appears not influenced by the variation of $\mathrm{I}_{\text {ads }}$. The average handover delay of dual-interface $\mathrm{MH}$ in the simulation is $39.9 \mathrm{~ms}$ for $\mathrm{HA}$ ÆFA movement, and $4.7 \mathrm{~ms}$ in the reverse direction.

\section{Conclusions}

In this paper, we have presented a multi-interface scheme for IEEE 802.21 media independent handover. The proposed scheme is compatible with transport protocols already in use, e.g. TCP and UDP, It works well with Mobile IPv4 without particular setting in agent routers. A dual-interface MH model has been designed and implemented in ns 2.29 to validate the proposed scheme. The comparison results (with single-interface $\mathrm{MH}$ ) show that dualinterface can effectively decrease data transmission break time and end-to-end packet delay in handover. Moreover, dual-interface can eliminate the influence of the advertisement interval on handover delay. This would make end-to-end packet delay independent of access domain in handover. Such a feature would especially favor NG heterogeneous networks that may be based on disparate technologies and run by multiple operators.

\section{References}

[1] F. André, J.-M. Bonnin, B. Deniau, K. Guillouard, N. Montavont, T. Noel, and L. Suciu, "Optimized Support of Multiple Wireless Interfaces within an IPv6 End-terminal," presented at Smart Objects Conference (SOC'2003), Grenoble, France, May 2003.

[2] C.-W. Ng and T. Ernst, "Multiple access interfaces for mobile nodes and networks," 2004.

[3] R. Chandra, P. Bahl, and P. Bahl, "MultiNet: connecting to multiple IEEE 802.11 networks using a single wireless card," March 2004.

[4] I. Ramani and S. Savage, "SyncScan: practical fast handoff for 802.11 infrastructure networks," presented at INFOCOM 2005. 24th Annual Joint Conference of the IEEE Computer and Communications Societies, Miami, FL, March 2005.

[5] IEEE 802.21, "IEEE 802.21 Media Independent Handover," vol. 2006: IEEE 802.21, pp. http://www.ieee802.org/21/.

[6] ns2, "The Network Simulator - ns-2," http://www.isi.edu/nsnam/ns/, 2006.

[7] C. Perkins, "RFC 3220 - IP Mobility Support for IPv4," IETF Network Working Group, January 2002.

[8] D. Johnson, C. Perkins, and J. Arkko, "RFC 3775 Mobility Support in IPv6," IETF Networking Group, June 2004.

[9] N. Montavont, R. Wakikawa, T. Ernst, C. Ng, and K. Kuladinithi, "Analysis of Multihoming in Mobile IPv6," in draft-ietf-monami6-mipv6-analysis-01: IETF MONAMI6 Working Group, June 26, 2006.

[10] J. Ylitalo, T. Jokikyyny, T. Kauppinen, A. J. Tuominen, and J. Laine, "Dynamic network interface selection in multihomed mobile hosts," presented at The 36th Annual Hawaii International Conference on System Sciences, Jan 2003.

[11] H. J. Wang, R. H. Katz, and J. Giese, "Policy-enabled handoffs across heterogeneous wireless networks," presented at The Second IEEE Workshop on Mobile Computing Systems and Applications (WMCSA), Feb 1999.

[12] Q. Song and A. Jamalipour, "Network selection in an integrated wireless LAN and UMTS environment using mathematical modeling and computing techniques," Wireless Communications, IEEE [see also IEEE Personal Communications], vol. 12, pp. 42, 2005.

[13] NOAH, "NO Ad-Hoc Routing Agent," http://icapeople.epfl.ch/widmer/uwb/ns-2/noah/.

[14] K. Pahlavan, P. Krishnamurthy, A. Hatami, M. Ylianttila, J. P. Makela, R. Pichna, and J. Vallstron, "Handoff in hybrid mobile data networks," Personal Communications, IEEE, vol. 7, pp. 34, 2000. 